\title{
Application of robots LEGO Mindstorms Education EV3 in the learning process of specialty Mechatronics
}

\author{
[Stoychev M., Kartunov S., Rachev P.]
}

\begin{abstract}
This article contains information about the course "Robotic modules and systems in production" for specialty Mechatronics in TU of Gabrovo, Bulgaria. Below are examples of robots that students develop exercises. Apply didactic approach that is very modern in training not only for students. With Lego Mindstorms easily can be developed a small model of industrial robot or a some system that is used in real life.
\end{abstract}

Keywords-Lego Mindstorms, specialty Mechatronics

\section{Introduction}

Lego Mindstorms is a series of kits which contains software and hardware for developing and programming a different robots. The main part of every set is the Lego Mindstorms programmable brick (programmable logic controller) thatruns different kinds of programs. The brick manage many sensors and motors which has a specific functions.

Untill now Lego Mindstorms has a three generations, as the main difference between them is the programmable brick. The first generation called RCX contains two motors, two touch sensors, and one light sensor. The second generation of Mindstorms is called NXT and contains: 3 servo motors, 4 sensors (ultrasonic, sound, touch, and light). With Lego Mindstorms easily can be developed a small model of industrial robot or a some system that is used in real life.

\section{Exposition}

Lego Mindstorms EV3 is officially released in stores on September 1, 2013. It comes in two versions - Home Set and Education Set. It is the third generations of the Mindstorms series. The "EV" meant "evolution" and the " 3 " is the number of the generation of the set. The biggest difference between NXT and EV3 is in the CPU of the programmable brick. EV3 has a more powerful ARM9 CPU running Linux. Also USB connector and Micro SD slot (up to 32GB) $[1,2]$.

The EV3 Home (31313) set contains: 1 EV3 programmable brick, 2 Large Motors, 1 Medium Motor, 1 Touch Sensor, 1 Color Sensor, 1 Infrared Sensor, 1 Remote Control, cables, USB cable, and 585 TECHNIC elements.

The Education EV3 Core Set (45544) set contains: 1 EV3 programmable brick, 2 Large Motors, 1 Medium Motor, 2 Touch Sensors, 1 Color Sensor, 1 Gyroscopic Sensor, 1 Ultrasonic Sensor, cables, USB cable, 1 Rechargeable battery and many TECHNIC elements. The Education EV3 Core Set can be expanded with an Expansion Set (45560) which has a 853 more LEGO elements and gave the possibility for building more complicated systems.

With the EV3 Education Core Set we can build 5 different robots - Robot Arm, Puppy, Gyro Boy, Educator and Color Sorter. If we add the Expansion set we can build 5 more robots - Tank bot, Stairclimber, Znap, Remote control and Elephant. And of we have 2 Core Sets an 1 Expansion Set there is a possibility for building one big robot called Spinner Factory.

Fig. 1. Examples of robots

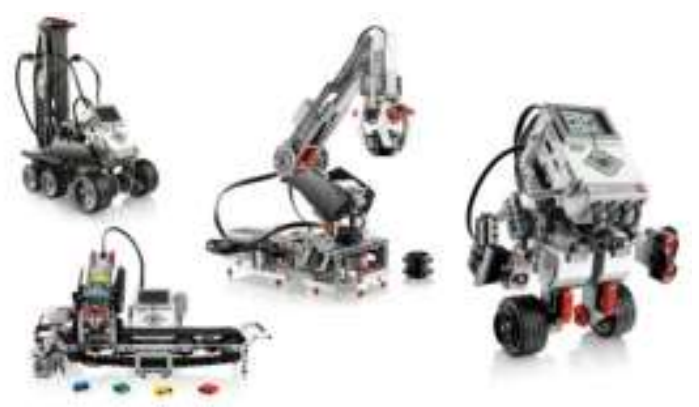

Let's have a closer look to the robots Stairclimber, Gyro Boy, Color Sorter and Robot Arm.

\section{Stairclimber}

For building the Stairclimber are needed two EV3 Sets - Education Core Set (45544) and Expansion Set (45560). The main function of the robot is to climb stairs. But there is a certain size of the stairs that the robot can handle - the maximum height of a single step have to be $17,5 \mathrm{~cm}(6,8 \mathrm{in})$ and the minimum width of a single step $26 \mathrm{~cm}$ (10,2 in).

The robot consists of two main parts. Body where the EV3 Brick is located and rear movable part whereby a 1 Large motor driving a chain, the body 
Fig. 2. Stairclimber-robot

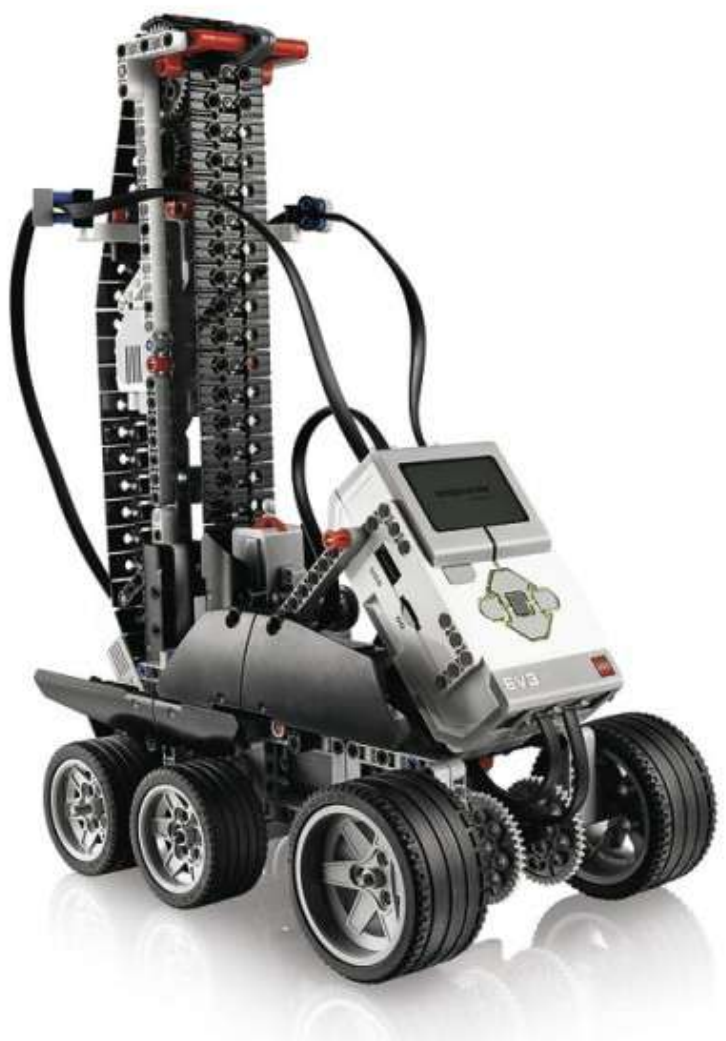

moves up wardsover the stairs. In the whole construction there are: 2 Large motors, 1 Medium Motor, 1 Touch Sensor and 1 Gyro sensor. The robot has a 3 axis - the front one is driven by a large motor, the rear one - by a medium motor and the middle axis it is not driven by a motor, but there is located a brake mechanism which does not allows the robot to drive backwards. For developing of program for the Stairclimber is used the software Lego Mindstorms EV3 Home Edition. It is a ROBOLAB GUI-based programming software, developed at Tufts University using the National Instruments Lab VIEW as an engine.

The program performs the following functions: first the gyro sensor resets itself, then the robot moves horizontaly. It moves like that until the gyro sensor change it's value to 8 or more degrees. The motor of the rear axis stops and the large motor which drives the chain starts. The whole body moves upwards over the step until a touch sensor on the top of the rear movable part switch off the motor of the chain. Then the robot moves horizontaly untill the rear movable part touch the step. The motor of the chain starts and brings back rear movable parts in starting position. The whole robot is over the step and the gyro sensor reset itself again and the cycle repeats again and again depends on that how many steps there are. The program can be written to be an endless cycle or for certain number of steps.

Fig. 3. Gyro Boy (Balancing Robot)

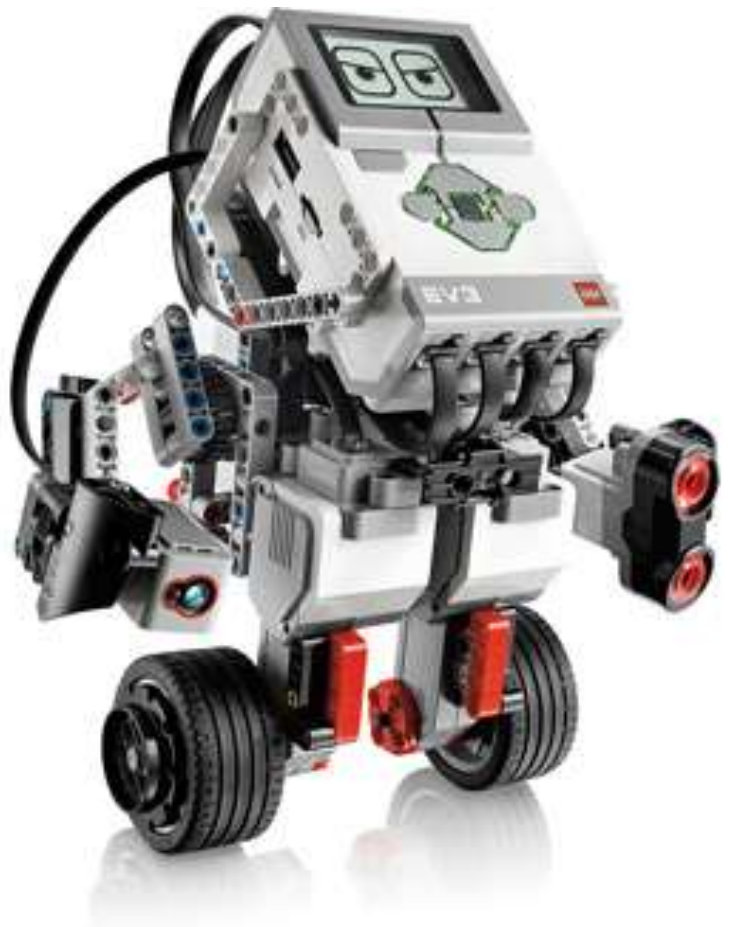

Gyro Boy (Balancing Robot)

For the realization of the Gyro Boy is needed only the Core Set (45544). The robot looks like a humanoidas the body is build from two large motors with wheels. Between them is located a gyro sensor. On the top is located the EV3 controller, in the back it has a medium motors for hand movements. On the both hands has a different sensors - color sensor and Ultrasonic sensor. The main function of the robot is to keep balance on its two wheels without any help.

To be that possible the robot have to start from the ideal vertical position. He is placed on a special stand that grants an ideal vertical position.

The program consists of 3 main parts: 1calibrating; 2- balance cycle; 3 - drive control. The first part runs when the robot is still on the stand. It reads the values and memorize them. Then the robot is moving out of the stand and in the balance cycle compares the moment values with that from the first part of the program. That is how the balance working - the program is trying to stick with the values from calibrating. In the drive control the robot can be managed by the sensors located on the both hands. For example using the ultrasonic sensor the robot can move forward and avoid obstacles or using the color sensor can make different moves for every different colors. 
Fig. 4. Color Sorter

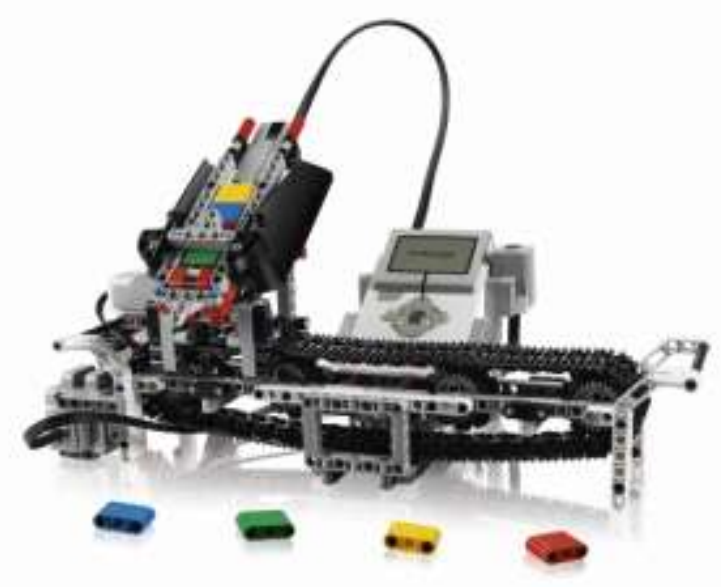

Color Sorter robot

For developing of the Color Sorter we need only the EV3 Core Set (45544). The robot is horizontally orientated. It has a movable part which drops the color bricks. This parts is moved by a large motor and chain. In the left end of the chain is located a touch sensor and next to the EV3 brick there is a color sensor. The robot works by the following way: first the operator shows a color brick to the color sensor. The program memorize the order of color bricks. The maximum bricks that can have the movable part is 8 . After the memorizing cycle is over the program reads the order and drops the brick to their own place (from left to right - blue - green - yellow - red). After every drop the movable part goes to the left end and presses the touch sensor. That how the EV3 brick know when to move forward in the program. After the last brick is droped the program ends.

\section{Robot Arm}

The Robot Arm builded from EV3 Mindstorms is a small model of a real industrial robot (manipulator). Here we need only the Core Set (45544) of the Mindstorms EV3 Education.

The main function of the robot is to move objects while they've been handled by the arm.

The construction consists of: 2 large motors; 1 medium motors and 2 touch sensors. The body of the robot is builded from the EV3 Brick and a small construction which is the base of the rotating arm. On the bottom is located 1 large motor who rotates the arm. This motor is managed by a touch sensor. On the arm itself is located second large motor for the translation move on the vertical axis. In the end of the arm is located a grabing device which is driven by a medium motor. The robot can be programmed to runs itself for the same moves every time with a certain number of objects or can be managed by a Android device connected via Bluetooth operating by a human.

Fig. 5. Robot Arm

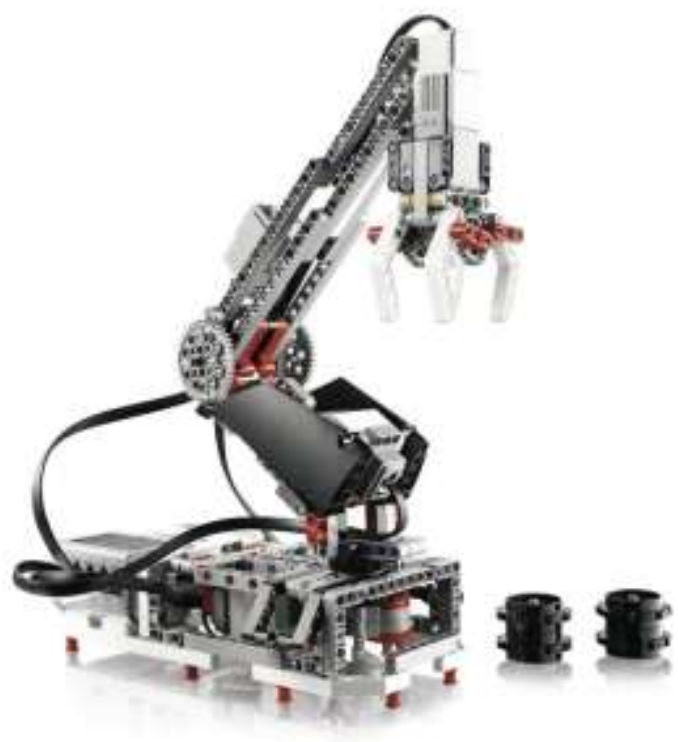

\section{Conclusion}

Apply didactic approach that is very modern in the learning process for students of specialty Mechatronics. With Lego Mindstorms easily can be developed a small model of industrial robot or a some system that is used in real life. These options are very diverse.

\section{REFERENCES:}

[1] www.Lego.com

[2] www.Robotsquare.com

\section{About the authors}

Prof. Dr-Ing. Stefan Kartunov, TU Gabrovo, Bulgaria, Hadji Dimitar Str. 4, Department MU, Mechanical Engineering, E-mail: Tel.++35966827365, Lab. Micro- and Nanotechnologies

Research interests: Technological process of micro- and nano elements, CAD/CAM/CAEstudies, simulations and animations before/in the production

Ass. Prof. Dr-Ing. Petar Rachev, TU Gabrovo, Bulgaria, Hadji Dimitar Str. 4, Department MU, Mechanical Engineering, E-mail: Tel.++35966827365

Student Ing. Mariyan Stoychev, Technical University of Gabrovo, Bulgaria, Hadji Dimitar Str. 4, Department MU, Mechatronic 